\title{
Complexity of Gender Dependent Contributing Factors on the Number of Lifetime Sexual Partners among Smartphone Application Users: A Path Analysis Model
}

Nooshin Zarei

Shiraz University of Medical Sciences

Elahe Khaksar

Shiraz University of Medical Sciences

Fatemeh Roosta

Shiraz University of Medical Sciences

Hassan Joulaei ( $\square$ joulaei_h@yahoo.com)

Shiraz University of Medical Sciences https://orcid.org/0000-0002-8498-5331

\section{Research article}

Keywords: Smartphone applications, premarital relationships, sexual partner, attitude, path analysis

Posted Date: December 4th, 2019

DOI: https://doi.org/10.21203/rs.2.18180/v1

License: (c) (1) This work is licensed under a Creative Commons Attribution 4.0 International License.

Read Full License 


\section{Abstract}

Background Smartphone apps provide a more accessible way for people to find friends and potential sexual partners. This study aimed to investigate the effects of contributing factors on the number of lifetime sexual partners and its gender differences among smartphone application users.

Methods This cross-sectional study was conducted in the summer of 2017 in Southwest of Iran via an online web-based questionnaire. Out of 414 smartphone users who completed the questionnaire, 169 were excluded due to not using mobile or apps to access sexual information and 245 were finally studied. Data were analyzed using SPSS software version 25.0 and Mplus version 6.12. The path analysis was run for both men and women separately. The significant level was considered to be $<0.05$ in all analysis. In addition, goodness of fit models were used by determining several indices.

Results For both genders, the most effective variables on the number of lifetime sexual partners was the attitude toward the premarital relationship and more permissive attitude was accompanied with more number of sexual partner. Other similar contributing factors in both genders were calling by the Internet, duration of the Internet usage, marital relationship, employment status, birth place, emotional instability, and openness to experience personality. Intimacy with parents was another effective factor, which was only effective for females.

Conclusion Due to the necessity of using smartphones, pre-puberty training and informing about the potential risks of the virtual environment should be considered. In this way, the role of parents should not be ignored.

\section{Background}

There has been a dramatic rise over the past two years in the percentage of people who use the Internet and own a smartphone[1]. According to the Internet world statistics, internet users per 100 person were 0.9 in 2000 and increased to 39.4 in 2014 [2]. Reports illustrated that Iran is among the top 20 countries with the highest number of Internet users. The number of Internet users was 250,000 in Dec, 2000 that reached 62,702,731 in Dec, 2018 (76.0\% penetration) [3]. Along with the growing popularity of smartphones, a variety of location-based social networking applications have been launched. These apps provide an easier way for people to meet potential partners through identifying nearby users, which might facilitate sexual activities because people can easily find sexual partners $[4,5]$.

Exposure to more sexually explicit contents was associated with more permissive attitudes toward premarital sex and stronger endorsement of the sexual standards[6]. As a result, some dysfunctions like engagement in sexual behaviors occur[7, 8]. A study on 944 Iranian high school students (2019) showed that $71.7 \%$ of the teenagers had their own cell phone and use it every day, of whom $49.8 \%$ had opposite sex partners and $23.1 \%$ met each other in a vacant house, $34.7 \%$ of them received sexts in the past 6 months. Students who sent and received sext were more likely to report having a partner of the opposite sex and engaging in sexual activity $(p<0.001)[8]$. 
Gender difference is also identified in premarital sexual attitudes and behaviors, with more permissive attitudes toward premarital sex among males[9]. A study in Iran showed that males significantly had more liberal attitudes than females in their acceptance of premarital sex. Being a male, of a younger age, single, and less religious were important determinants of a liberal sexual attitude[10]. Moreover, males had more access to the Internet and more engagement in high-risk sexual behaviors. Internet pornography consumption was also positively associated with having sex with multiple partners and having extramarital sex [1,11-13]. Moreover, education, higher income, shyness, and loneliness were related to more use of the Internet or own a smartphone $[1,14,15]$. Personality traits were also related to risky behaviors[16]. A study in Africa showed that vulnerable personality and behavioral attributes are related to association with deviant peers, which is related to risky sexual behavior[17]. Therefore, this study aimed at estimating the effects of some contributing factors on the number of lifetime sexual partners and its gender differences among smartphone application users who accessed sexual contents.

\section{Methods}

\section{Subjects and sampling}

This was a cross-sectional study. The measurement tool was a web-based structured questionnaire designed by a software engineer which was available online. The study population was smartphone users in Fars province of Iran. 414 adults in the summer of 2017 completed the questionnaire. Inclusion criteria were having a smartphone, connecting to the internet, utilizing mobile or apps to access sexual information, completing an informed consent form at the end of the online questionnaire and age 18 to 40 years. One-hundred and sixty nine smartphone users were excluded due to not using mobile or apps to access sexual contents and 245 were finally studied.

Given the sensitivity of the subject and nature of the studied population that must be smartphone users, we made a virtual announcement. For this purpose, we provided an access for the participants to complete the questionnaire through the university websites and other virtual sites like Instagram, WhatsApp, Telegram channels, and groups.

\section{Study instrument}

We considered demographic variables - gender, age, place of birth (capital city, small city, and rural areas), education, marital status, occupational status, and social class. We also measured the Internet usage as a self-report item on a six-point scale with response choices as follows: 1) less than $1 \mathrm{~h}$ per week, 2) 1 to $5 \mathrm{~h}$ per week, 3) less than $1 \mathrm{~h}$ per day, 4) 1 to $3 \mathrm{~h}$ per day, 5) 3 to $5 \mathrm{~h}$ per day, and 6) more than $5 \mathrm{~h}$ per day. To determine the types of Internet usage, we used the internet-service scale with 13 items in a 5 -point Likert-type ranging from $1=$ never to $5=$ very often. The respondents were asked to indicate how often they used different functions of smartphones through the Internet for different purposes including communication (E-mail and Chat), leisure (music, role-playing, shopping), and 
academic (research, course participation on-line) $[18,19]$. In order to determine the rate of using mobile apps, the amount of using different types of mobile apps-such as what's app, Telegram, Instagram, Imo, Line, Tango, Beetalk, Skype, etc.--was inquired.

A validated Portuguese version (Neto 2014) of the revised UCLA Loneliness Scale was used to assess loneliness. All the 6 items were scored on a 4-point scale ranging from 1 (never) to 4 (often). Higher scores indicate greater loneliness [20].

Adolescents' Attitude towards Premarital Sex Questionnaire (AATPSQ) developed by Nwankwo (1997) was used to assess the participants' attitude. The questionnaire consisted of 21 items in 7-point Likerttype scale from 1 (strongly disagree) to 7 (strongly agree)[21]. Due to the social stigma of discussions about sexual relationships and limitation of the ethics committee of the project, we omitted 5 questions. The Cronbach's alpha for the attitude scale was 0.913. Ten-Item Personality Inventory (TIPI) was used to measure the Big-Five personality domains (Extraversion, agreeableness, conscientiousness, emotional stability, openness to experiences). The respondents asked to write a number next to each statement to indicate the extent to which they agree or disagree with that statement. You should rate the extent to which the pair of traits applies to them (Score 1 for strongly Disagree, score 7 for strongly Agree)[22]. In addition, the participants were asked to answer two 5-point-Likert questions about their religious beliefs.

\section{Data Analysis}

For statistics analysis, IBM SPSS version 25.0 and Mplus version 6.12 were used. At first we selected variables that had significant correlation with the number of lifetime sexual partners, attitude towards premarital relationships, and the personality trait of openness to experiences with $p$-value $<0.2$. The path analysis was run for both men and women because mean of them were significant with the number of lifetime sexual partners with t-test to express a direct and indirect relationships in explaining the number of lifetime sexual partners. A standardized Path coefficient (beta) showed the direct and/or indirect effect of independent and intermediate dependent variables on a final dependent variable in the model.

In the current study, independent variables were birth place, marital status, occupational status, duration of the connection to the Internet, the personality trait of openness to experiences, intimate relationship with parents, agreeableness, consciousness, emotional stability, Internet call and attitude towards premarital relationships.

The significant level was considered to be $<0.05$ in all analysis. In addition, goodness of fit models were used by determining several indices, including the root mean square error of approximation (RMSEA), Tucker-Lewis index (TLI), and comparative fit index (CFI). CFI and TLI values above 0.90 and RMSEA below 0.08 indicated the good fitness of the model.

\section{Results}


Out of 245 participants who were included in the study, 163 (66.5\%) were females and $82(33.5 \%)$ were males. Mean age of participants was $26.6 \pm 8.2$ year. Regardless of marital status, $16.7 \%$ of females and $36.5 \%$ of males declared having more than 2 sexual partners during their lifetime. Results of sociodemographic characteristics according to gender were shown in the table 1.

The mean duration of connecting to the Internet was $4.95 \pm 1.1$ hours a day and the majority of both males and females $(61.0 \%$ and $66.9 \%$, respectively) used the Internet more than 3 hours a day.

\section{Path analysis model}

The literature review showed gender difference in access to the Internet, engagement in high-risk sexual behaviors, the attitude, and some other factors. Besides, our results showed that the number of lifetime sexual partners was significantly different according to gender $(p=0.001)$. Therefore, the path analysis was conducted separately for males and females. The effective variables on the number of lifetime sexual partners have shown for both men and women (Fig 1 and 2).

Among female, attitude toward pre-marital relationship $(\beta=0.561)$ had the most direct effect on the number of lifetime sexual partners with more number of partner amongst women who had more liberal attitude. Marital relationship and being employed affect both directly $(\beta=0.383$ and $\beta=0.278$, respectively) and indirectly ( $\beta=0.213$ and $\beta=0.055$, respectively). In addition, have an openness to experience personality $(\beta=0.248)$, less intimacy with parents $(\beta=-0.222)$, less emotional stability $(\beta=-$ $0.202)$, and birth in large cities $(\beta=0.186)$ had direct effect on the number of lifetime sexual partners, while, the effect of calling by the Internet $(\beta=0.188)$ and duration of the Internet usage $(\beta=0.158)$ were only indirectly (Figure 1). The RMSEA of this model was 0.04 and CFI and TLI were 0.986 and 0.946 , respectively.

For male group, attitude toward pre-marital relationship was also the most effective factor on the number of lifetime sexual partners $(\beta=0.583)$ with a direct and indirect effect through openness to experience personality. Moreover, more Internet call $(\beta=0.260)$, openness to experience personality $(\beta=0.248)$, birth in a larger city $(\beta=0.190)$, longer duration of the Internet usage $(\beta=0.178)$, and marital relationship ( $\beta=$ 0.158 ) affected directly on more number of lifetime sexual partners, while being employed and emotional stability factor had both direct and indirect effect (Figure 2). The RMSEA of this model was 0.074 and CFI and TLI were 0.98 and 0.936 , respectively.

A comparison showed that all the effective factors were similar in both genders, but intimacy with parents was only effective for female which reversely affected number of lifetime sexual partners.

\section{Discussion}

This study aimed to investigate the effects of some contributing factors on the number of lifetime sexual partners and its gender differences among smartphone application users who accessed sexual contents. 
414 adults completed the questionnaire and 245 individual who had access to sexual contents were finally studied, which shows the prominent prevalence of using such contents among Iranian adults. Similarly, a study in Indonesia as another Muslim country, showed that pornography is as widely and readily consumed and is comparable international studies predominantly utilizing Western background samples [23].

More than 60 percent of both genders used the Internet more than 3 hours per day which confirmed the report that showed Iran is among the top 20 countries with the highest number of the Internet users [24]. Despite the fact that premarital sexual intercourse and even talking about sexual issues in the cultural context of Iran is still a taboo, $16.7 \%$ of females and $36.5 \%$ of males declared having more than 2 sexual partners during their lifetime which is in accordance with previous studies $[25,26]$. In India, as a country with a similar cultural context, 39 percent of the male college students reported having experienced sex before marriage despite religious and cultural limitations[27].

The most effective factor of more number of sexual partners for both gender was more liberal attitude toward pre-marital relationship. Other similar contributing factors in both genders were more calling by the Internet, longer duration of the Internet usage, marital relationship, being employed, birth in large cities, less emotional stability, and having openness to experience personality. Intimacy with parents was another effective factor, which was only effective for females.

For both genders, the attitude toward premarital relationship directly and indirectly affected the number of partners and more liberal attitude was associated with openness to experience personality, which leads to more number of lifetime sexual partners. While it was an independent factor amongst males, it was a mediator factor among females, which was affected by longer time of the internet call, longer duration of the internet usage, and being single. However, longer internet call, more duration of the internet usage among males affect number of lifetime sexual partners directly.

According to marital status, in both genders, divorced users had more number of partners than others, and married individuals also had more partner in compare with single ones. However, a difference was a direct effect of this factor among males and both direct and indirect effect of being single through increasing liberal attitude among females. A study in India also showed that single people have experienced more premarital relationships than married ones[27]; this seems to be in contrast with the current result. The difference can be due to the distinction of the present study with previous ones. We consider the number of lifetime sexual partners rather than just having a premarital sexual experience, per se. Thus, this conclusion is logical. Given that in the path analysis, all variables were recoded into twodimensional ones, widowed and divorced people were considered as married and the average number of partners was higher for them. In addition, the findings of this study showed that married people have more conservative attitude. Hence, if a person has more than one sexual partner during his lifetime, it seems that this partner is related to his single years.

Being employed was another effective factor with a direct effect on number of lifetime partners, besides, it had also an indirect effect through openness to experience personality in both genders. Due to the high 
prevalence of unemployment in Iran, employed people are wealthier. A meta-analysis study (2015) also confirmed that high economic status is associated with high-risk sex[28].

The effect of emotional stability was somewhat different according to gender. Among females, this factor had only a direct effect and a woman with emotional instability had more partners, while the instability in male increase the number of partners directly and indirectly through effect on openness to experience character. Previous findings also indicate that personality type is effective and having a vulnerable personality increases the chance of engagement in high-risk sexual behaviors $[15,17]$. In addition, adults using the Internet for pornography have had different social characters compared to those who used the internet for information[29].

Moreover, birth in a larger cities had a direct effect and increase the number of life time sexual partners in both genders. This is in line with previous studies that showed the living in urban centers affects the experience of premarital relationships and high risk sex $[27,28]$.

Intimacy with parents was another effective factor, just in females, with only a direct effect on the number of sexual partners. Therefore, the number of sexual partners was lower for female smartphone users who had more intimate relationship with their parents. This is in accordance with previous studies that poor relationship with parents plays an important role in the continuity of risky behaviors and romantic relational aggression and victimization[29].

To the best of our knowledge this is the first study to estimate the effects of psychosocial factors on the number of lifetime sexual partners among smartphone application users in Iran as a Muslim country. Another novelty of this research was use of a web-based structured questionnaire which decreased the bias and assured participants that their identity would not be disclosed. However, due to cultural barriers we were unable to announce for the online survey and as a result, we could not include more people in the study.

\section{Conclusion}

In both genders, the attitude towards premarital relationships was the most effective factor of the number of lifetime sexual partners in both direct and indirect ways. As more liberal attitude increases the likelihood of engaging in high-risk sexual behaviors and more number of sexual partners, sex education should be noticed to reduce mental, physical, and medical damage. Moreover, as longer time of calling by the internet, duration of the Internet usage, and being single were accompanied with more permissive attitudes and number of lifetime partners among females, increasing awareness of single females in order to better use of the Internet and fill their leisure time with beneficial activities should be considered.

Besides, due to the inevitable use of smartphones in the present era, pre-puberty training and informing about the potential risks of using the virtual environment should be taken into consideration. In addition, in order to control the effect of the personality trait of openness to experience and prevent the 
involvement of these individuals in high-risk behaviors, it is important to identify them and consider the education and care they need.

\section{List Of Abbreviations}

AATPSQ:Adolescents' Attitude towards Premarital Sex Questionnaire; TIPI: Ten-Item Personality Inventory; TLI: Tucker-Lewis index; CFI: comparative fit index.

\section{Declarations}

\section{Ethics approval and consent to participate}

To minimize the missing data, the questionnaire was designed in such a way that only two questions could not be answered. However, we asked the respondent to click the registration button at the end of the questionnaire if they were satisfied to record the information. Ethical approval was obtained from the Ethics Committee of Shiraz University of Medical Sciences (No. 12385).

\section{Consent for publication}

Not applicable

\section{Availability of data and materials}

The datasets generated and analyzed during the current study are not publicly available due to privacy restrictions. Respondents were informed during the consent process that the data would be available only to the Shiraz University of Medical Sciences.

\section{Competing interests}

The authors declared no competing interests.

\section{Funding}

Research Deputy of Shiraz University of Medical Sciences financially supported the project (No. 12385).

\section{Authors' contributions}

NZ contributed to the conception and design of the study and data collection. NZ, EK, and FR did the statistical analysis. NZ, HJ, EK drafted the original manuscript. All authors read and approved the final manuscript. 


\section{Acknowledgements}

The authors give their gratitude to the Research Deputy of Shiraz University of Medical Sciences regarding their financial support, to Dr. Parvin Afsar Kazerooni and Dr. Mojgan Sabet due to coordination with university security and informing the studied population. We also appreciated Dr. Nasrin Shokrpour who edited this manuscript.

\section{References}

1.Poushter J: Smartphone Ownership and Internet Usage Continues to Climb in Emerging Economies. Pew Research Center: Global Attitudes \& Trends 2016.

2.Internet world stats. Top 20 countries with the highest number of internet users. 2015. Retrieved from http://www.internetworldstats.com/top20.htm. .

3. Internet world stats. Top 20 countries with the highest number of internet users. 2019. Retrieved from https://www.internetworldstats.com/top20.htm. 2019.

4.Choi E, Wong J, Fong D: The use of social networking applications of smartphone and associated sexual risks in lesbian, gay, bisexual, and transgender populations: A systematic review. AIDS care 2017, 29(2):145-155.

5.Choi EP-H, Wong JY-H, Lo HH-M, Wong W, Chio JH-M, Fong DY-T: The impacts of using smartphone dating applications on sexual risk behaviours in college students in Hong Kong. PLoS one 2016, 11(11):e0165394.

6.Zhang Y, Miller LE, Harrison K: The relationship between exposure to sexual music videos and young adults' sexual attitudes. Journal of Broadcasting \& Electronic Media 2008, 52(3):368-386.

7. Billieux J, Philippot P, Schmid C, Maurage P, De Mol J, Van der Linden M: Is dysfunctional use of the mobile phone a behavioural addiction? confronting symptom-based versus process-based approaches. Clinical psychology \& psychotherapy 2015, 22(5):460-468.

8.Ghorashi Z: Teenage Sexting and Sexual Behaviors in an Iranian Setting. Sexuality \& Culture 2019:1-9.

9.Zuo X, Lou C, Gao E, Cheng Y, Niu H, Zabin LS: Gender differences in adolescent premarital sexual permissiveness in three Asian cities: effects of gender-role attitudes. Journal of Adolescent Health 2012, 50(3):S18-S25.

10.Motamedi M, Merghati-Khoei E, Shahbazi M, Rahimi-Naghani S, Salehi M, Karimi M, Hajebi A, Khalajabadi-Farahani F: Paradoxical attitudes toward premarital dating and sexual encounters in Tehran, Iran: a cross-sectional study. Reproductive health 2016, 13(1):102. 
11.Li N, Kirkup G: Gender and cultural differences in Internet use: A study of China and the UK. Computers \& Education 2007, 48(2):301-317.

12.Jones BH, Chin AG, Aiken P: Risky business: Students and smartphones. TechTrends 2014, 58(6):7383.

13.Wright PJ, Randall AK: Internet pornography exposure and risky sexual behavior among adult males in the United States. Computers in Human Behavior 2012, 28(4):1410-1416.

14.Tossell CC, Kortum P, Shepard C, Rahmati A, Zhong L: You can lead a horse to water but you cannot make him learn: Smartphone use in higher education. British Journal of Educational Technology 2015, 46(4):713-724.

15.Bian M, Leung L: Linking loneliness, shyness, smartphone addiction symptoms, and patterns of smartphone use to social capital. Social Science Computer Review 2015, 33(1):61-79.

16.Cooper ML: Toward a personx situation model of sexual risk-taking behaviors: Illuminating the conditional effects of traits across sexual situations and relationship contexts. Journal of Personality and Social Psychology 2010, 98(2):319.

17.Brook DW, Morojele NK, Zhang C, Brook JS: South African adolescents: pathways to risky sexual behavior. AIDS education and prevention 2006, 18(3):259.

18.Landers RN, Lounsbury JW: An investigation of Big Five and narrow personality traits in relation to Internet usage. Computers in Human Behavior 2006, 22(2):283-293.

19. Hamburger YA, Ben-Artzi E: The relationship between extraversion and neuroticism and the different uses of the Internet. Computers in Human Behavior 2000, 16(4):441-449.

20.Neto F: Psychometric analysis of the short-form UCLA Loneliness Scale (ULS-6) in older adults. European journal of ageing 2014, 11(4):313-319.

21.Eze IR: Adolescents' Attitude Towards Premarital Sex. Mediterranean Journal of Social Sciences 2014, 5(10):491.

22.Gosling SD, Rentfrow PJ, Swann Jr WB: A very brief measure of the Big-Five personality domains. Journal of Research in personality 2003, 37(6):504-528.

23.Hald GM, Mulya TW: Pornography consumption and non-marital sexual behaviour in a sample of young Indonesian university students. Culture, Health \& Sexuality 2013, 15(8):981-996.

24. The Statistics Portal. Number of smartphone users worldwide from 2014 to 2019 (in millions). Retrieved from http://www.statista.com/statistics/330695/number-of-smartphone-users-worldwide/ 
25.Shirpak KR, Chinichian M, Maticka-Tyndale E, Ardebili HE, Pourreza A, Ramenzankhani A: A qualitative assessment of the sex education needs of married Iranian women. Sexuality \& Culture 2008, 12(3):133150.

26.Roudsari RL, Javadnoori M, Hasanpour M, Hazavehei SMM, Taghipour A: Socio-cultural challenges to sexual health education for female adolescents in Iran. Iranian journal of reproductive medicine 2013, 11(2):101.

27.Adhikari R, Tamang J: Premarital sexual behavior among male college students of Kathmandu, Nepal. BMC public health 2009, 9(1):241.

28.Berhan Y, Berhan A: A meta-analysis of risky sexual behaviour among male youth in developing countries. AIDS research and treatment 2015, 2015.

29.Linder JR, Crick NR, Collins WA: Relational aggression and victimization in young adults' romantic relationships: Associations with perceptions of parent, peer, and romantic relationship quality. Social Development 2002, 11(1):69-86.

\section{Table}

Table 1: Socio-demographic characteristics of the studied sample according to gender 


\begin{tabular}{|c|c|c|c|}
\hline Variable & $\begin{array}{c}\text { Female } \\
\mathbf{N}(\%)\end{array}$ & $\begin{array}{l}\text { Male } \\
\text { N(\%) }\end{array}$ & P-value \\
\hline Educational level & & & 0.274 \\
\hline High school & $12(7.4)$ & $8(9.8)$ & \\
\hline Diploma & $26(16)$ & $18(22)$ & \\
\hline associate degree & $22(13.5)$ & 16(19.5) & \\
\hline University degree & $102(62.6)$ & $40(48.8)$ & \\
\hline Occupational status & & & 0.025 \\
\hline Full-time & $53(32.5)$ & $39(47.6)$ & \\
\hline Part-time job & $12(7.4)$ & $9(11)$ & \\
\hline Unemployed & $34(20.9)$ & $5(6.1)$ & \\
\hline Student & $64(39.3)$ & $28(34.1)$ & \\
\hline Marital status & & & 0.153 \\
\hline Single & 96(58.9) & $50(61)$ & \\
\hline Married & $67(41.1)$ & $32(39)$ & \\
\hline Birth place & & & 0.028 \\
\hline Capital city & $109(66.8)$ & $47(57.3)$ & \\
\hline Small city & $40(24.5)$ & $21(25.6)$ & \\
\hline Rural area & $14(8.6)$ & $14(17.1)$ & \\
\hline Calling by the Internet & & & 0.002 \\
\hline None & $57(40.4)$ & $38(46.4)$ & \\
\hline Low & $72(44.2)$ & $26(31.7)$ & \\
\hline Medium & $22(13.5)$ & $12(14.6)$ & \\
\hline High & $12(7.3)$ & $6(7.3)$ & \\
\hline Duration of connecting to the Internet & & & 0.011 \\
\hline less than $1 \mathrm{~h}$ per week & $1(0.6)$ & $1(1.2)$ & \\
\hline 1 to $5 \mathrm{~h}$ per week & $0(0)$ & $6(7.3)$ & \\
\hline less than $1 \mathrm{~h}$ per day & $6(3.7)$ & $4(4.9)$ & \\
\hline 1 to $3 \mathrm{~h}$ per day & $47(28.8)$ & 21(25.6) & \\
\hline More than $3 \mathrm{~h}$ per day & $109(66.9)$ & $50(61.0)$ & \\
\hline Intimate relationship with parents & & & 0.070 \\
\hline Strongly disagree & $59(36.2)$ & $31(37.8)$ & \\
\hline Disagree & $50(30.7)$ & $20(24.4)$ & \\
\hline Medium & $37(22.7)$ & 24(29.3) & \\
\hline Agree & $12(7.4)$ & $6(7.3)$ & \\
\hline Strongly Agree & $5(3.1)$ & $1(1.2)$ & \\
\hline Loneliness & $14.82 \pm 4.49$ & $14.72 \pm 4.20$ & 0.046 \\
\hline Agreeableness & $10.34 \pm 2.36$ & $10.11 \pm 2.24$ & 0.015 \\
\hline Extraversion & $7.36 \pm 2.72$ & $7.92 \pm 2.48$ & 0.004 \\
\hline Consciousness & $10.92 \pm 2.54$ & $10.59 \pm 2.44$ & 0.122 \\
\hline Openness to experiences* & $8.17 \pm 2.51$ & $8.56 \pm 2.30$ & $<0.001$ \\
\hline Emotional stability* & $8.47 \pm 2.83$ & $8.79 \pm 2.71$ & 0.001 \\
\hline Attitude towards premarital relat & $37.46 \pm 17.98$ & $54.69 \pm 19.04$ & $<0.001$ \\
\hline Number of lifetime sexual partner* & $1.18 \pm 1.47$ & $2.05 \pm 2.10$ & 0.001 \\
\hline
\end{tabular}

Mean $\pm \mathrm{SD}^{*}$

\section{Figures}




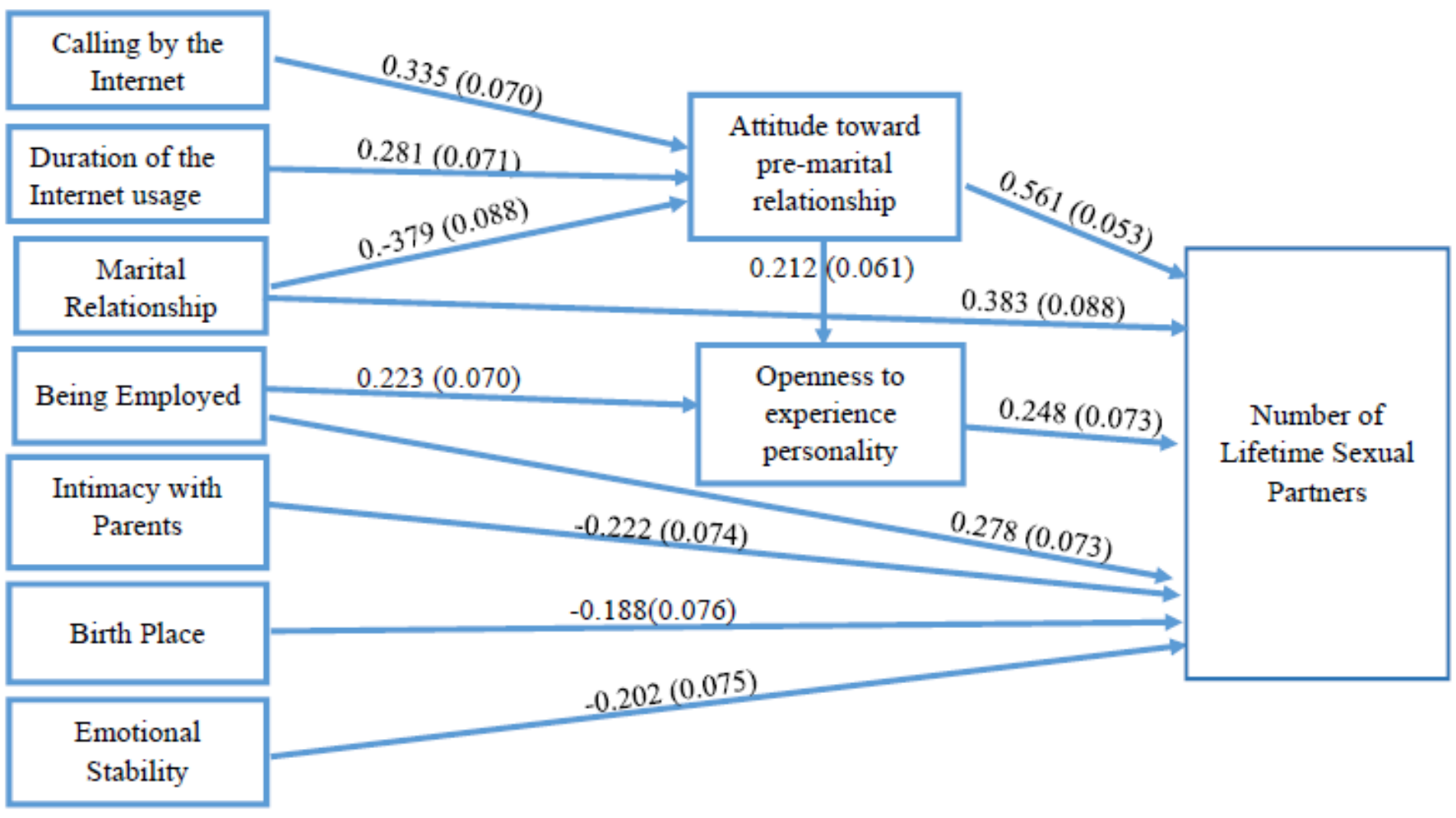

\section{Figure 1}

Path diagram of number of lifetime sexual partners among females

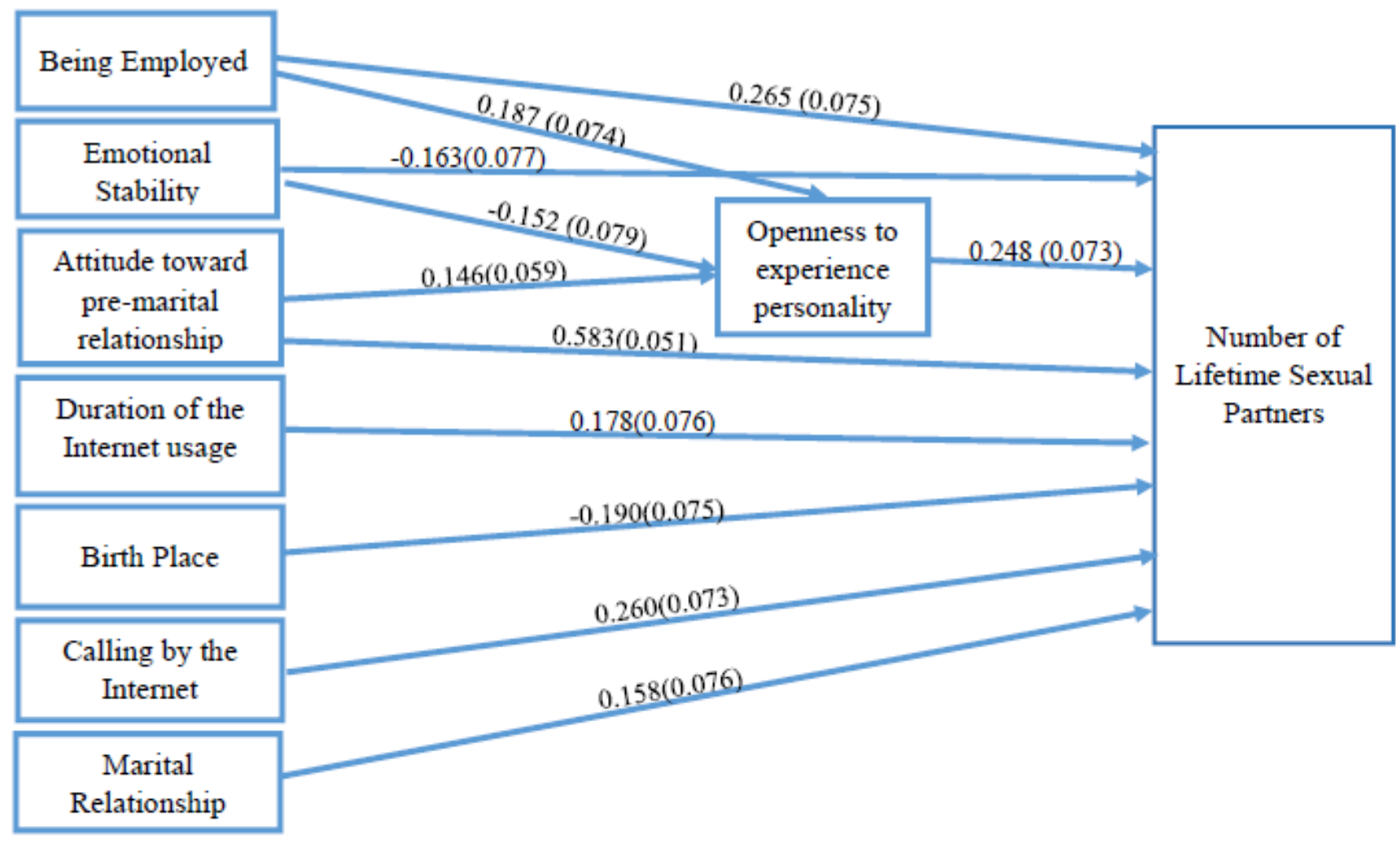


Figure 2

Path diagram of number of lifetime sexual partners among males *Numbers in the graphs represent standardize coefficient (standard error) 